\title{
ESTRUCTURAS DE PODER DE GÉNERO EN LAS UNIVERSIDADES DE NICARAGUA*
}

\author{
Mercedes Fernandez / Rebeca Centeno Orozco \\ Gilma Yadira Tinoco / Sandra Zúñiga \\ Comisión Interuniversitaria de \\ Estudios de Género (CIEG-Nicaragua) ${ }^{1}$
}

\section{El sistema sexo-género en Nicaragua}

os estudios de género han alcanzado en las últimas décadas, una diversificación disciplinaria y un alcance teórico e investigativo de tal relevancia, que han permitido fundamentar sólidamente la existencia de persistentes desigualdades sociales entre varones y mujeres, en todos los países del mundo. También en Nicaragua, se han llevado a cabo un buen número de estudios de calidad, que han puesto al descubierto la existencia de relaciones de poder sexo-génericas en diversas esferas de nuestra sociedad.

Al respecto, cabe destacar que según investigaciones económicas realizadas por la Fundación Internacional para el Desafío Económico (1997:3-5), las mujeres nicaragüenses presentan mayores tasas

* Ponencia presentada en el Encuentro de Universidades Latinoamericanas y del Caribe sobre Estudios de Género, celebrado el mes de agosto de 1999 en la Universidad de Panamá; y revisada para la Revista ANALISIS.

${ }^{1}$ Agradecemos la colaboración solidaria en la recolección de datos, de nuestra compañera de la CIEG-Nicaragua, Guiomar Talavera docente de la UCA, así como de nuestras estimadas colegas, las profesoras Lesbia Bermúdez (UNAN-Managua), Glenis Calderón (UNI) y Mayra Calero (UPOLI). 
de desempleo y subempleo que los varones. En el período de 19951996, de cada 100 mujeres económicamente activas, 85 tuvieron acceso a un empleo, frente al 91 por ciento de los hombres. Asimismo, la población femenina tiene una mayor presencia en sectores económicos de baja productividad y remuneración, como son los de comercio y servicios. Además, de cada 100 mujeres que trabajan, 75 pertenecen al sector informal, mientras que los varones están presentes en un 63 por ciento. En cuanto a las brechas de género en los ingresos a nivel nacional, las mujeres perciben un promedio mensual de un 26.3 por ciento inferior al ingreso promedio masculino.

El mismo estudio, revela desproporciones de género igualmente notables en el acceso al crédito. Mientras que del total de personas beneficiarias con préstamos, el 49.3 por ciento son mujeres, solamente reciben el 34.1 por ciento del monto total, fundamentalmente de fuentes crediticias informales o pertenecientes a Organizaciones No Gubernamentales (ONG's). Sin embargo, los varones son financiados básicamente por la banca privada, cuestión que está estrechamente relacionada con la mayor posibilidad de los hombres de garantizar la solicitud de préstamos mediante propiedades.

Tanto es así, que los datos hallados sobre la propiedad de la tierra, muestran disparidades aún más profundas entre varones y mujeres. Así, la citada investigación evidencia que del total de hogares que poseen títulos de tierra (32\%), solamente un 13 por ciento de éstos están en manos femeninas, representando un 9.8 por ciento del área total. Sin embargo, la propiedad masculina de la tierra constituye el 68 por ciento y corresponde al 80.2 por ciento del área total.

En cuanto al tiempo dedicado a las actividades de los ámbitos productivo y reproductivo, las mujeres son las encargadas del mayor tiempo invertido (85\%) en el espacio doméstico y los varones del menor (15\%). No obstante, en el espacio productivo, a las mujeres les corresponde el 47 por ciento del tiempo total y a los hombres el 53 por ciento. Sumando el tiempo dedicado en ambas esferas, se pone de manifiesto que las mujeres laboran más horas que los varones $(68 \%$ corresponde al trabajo femenino y el $32 \%$ al masculino).

Si nos introducimos en el orden de las relaciones familiares, un impactante estudio realizado en 1996, sobre la violencia conyugal en el Municipio de León, arroja que un 39 por ciento de mujeres ha sido golpeada por su marido o compañero alguna vez en su vida; una de cada cuatro mujeres que viven en pareja, estaba siendo maltratada en el período en el que se realizó el estudio. Además, una 
de cada tres mujeres maltratadas, lo ha sido durante el embarazo, y la mitad de ellas recibieron golpes en el vientre. Asimismo, una de cada tres fue obligada a tener relaciones sexuales durante la golpiza (Ellsberg et al., 1998:45).

\section{El sexismo y el androcentrismo en el conocimiento científico}

En las disciplinas científicas también se expresa la prevalencia del poder masculino, esta vez en el área del conocimiento. El modelo de ciencia hegemónico, únicamente ha considerado dignas de ser objeto y sujeto de conocimiento, las experiencias y las concepciones masculinas del mundo, suprimiendo o devaluando las prácticas y las formas de pensar diferenciadas de las mujeres. En correspondencia, la actividad científica se ha visto incapacitada a través de los siglos, para dar cuenta, tanto de la contribución de las mujeres a la vida social, como de la condición de subalternidad social de las mismas (Durán, 1982).

No obstante, los estudios de género realizados en las últimas décadas, han contribuido a develar el sexismo y el androcentrismo presentes en la tradición científica occidental. En primer lugar, desde el feminismo teórico, se han puesto en entredicho aquellas nociones androcéntricas positivistas de objetividad y racionalidad (Fox Keller, 1991; Haraway, 1995; Harding, 1996), que niegan el lado humano de la producción científica y la revisten de un carácter ahistórico, absoluto y neutral. De esta manera, están contribuyendo poderosamente a una transformación epistemológica, que coincide en buena medida y desde una vertiente propia, con diversas posiciones mantenidas por las nuevas filosofías de la ciencia (Brown, 1984), sobre la presencia de condicionamientos sociales, históricos e ideológicos en el quehacer científico.

Por otro lado, las investigaciones empíricas de género llevadas a cabo desde diversas disciplinas, han producido al menos otro efecto epistemológico relevante, como es la posibilidad de mostrar científicamente realidades de exclusión y subordinación de las mujeres en distintos contextos. En otras palabras, los estudios feministas constituyen una muestra fehaciente de una de las rupturas epistemológicas más contundentes con el positivismo científico; pero, no solamente en la manera de interpretar el conocimiento científico, sino también en el modo de construirlo, ya que han sido capaces de generar enriquecedoras miradas de la realidad que han 
visibilizado las relaciones y estructuras de poder sexo-genéricas, para lo cual han requerido plantear preguntas no neutrales, abrir nuevas líneas de investigación y utilizar metodologías innovadoras.

Esta labor no hubiese sido posible sin el compromiso ético-político que conlleva todo estudio feminista, en su búsqueda de alternativas, tanto de validación de los aportes femeninos a la sociedad reproductivos y productivos-, como de propuestas de participación y toma de decisiones de las mujeres en ámbitos sociales de los cuales han sido tradicionalmente relegadas. Por este compromiso no podemos ser autocomplacientes; precisamos una revisión permanente de la realidad de género en la que vivimos, a fin de identificar las limitaciones y posibilidades que tenemos en este proceso de transformación social.

Siendo la comunidad universitaria uno de los espacios fundamentales de producción científica y nuestro ámbito más inmediato de trabajo e incidencia, en ella se deberían percibir de algún modo, cambios efectivos en las relaciones entre varones y mujeres, como resultado de la inserción de los estudios de género en algunos centros de educación superior, así como consecuencia del trabajo realizado por el conjunto del movimiento de mujeres y feminista de Nicaragua.

\section{El poder de género en el sistema educativo}

La amplia literatura internacional sobre el tema que nos ocupa, ha posibilitado hacer evidente que las instituciones educativas formales no sean ajenas a los fenómenos de poder de género que permean la sociedad en su conjunto. Más bien, constituyen espacios de socialización privilegiados, en los cuales, desde edades tempranas, se construyen procesos de producción y reproducción de saberes, mentalidades y comportamientos que discriminan a las mujeres.

Esta potente facultad socializadora de la educación formal, pudiera convertirse en una herramienta idónea para la generación y consolidación de conocimientos no androcéntricos y de prácticas equitativas de género. Sin embargo, dicho potencial democrático no está siendo contemplado en las políticas educativas públicas de Nicaragua y, escasamente, en las propuestas a las mismas aportadas desde las organizaciones de la sociedad civil. En este contexto, en el que las condiciones de equidad de género no son vislumbradas como indicadores de calidad y democracia educativa, se torna más apremiante la necesidad de continuar explorando las múltiples 
formas de discriminación genérica en la labor educacional de nuestro país, así como de avanzar en el desarrollo de alternativas para su eliminación.

En este sentido, un esfuerzo absolutamente voluntario de las integrantes de la Comisión Interuniversitaria de Estudios de Género (CIEG-Nicaragua), ha hecho posible presentar en este nuevo Encuentro en Panamá2, un estudio preliminar que tiene como propósito general analizar las relaciones de poder de género en cuatro universidades de Nicaragua, en el período 1998-1999. Para ello se ha tomado en cuenta la distribución de hombres y mujeres en la matrícula y en las diversas áreas disciplinarias elegidas por el estudiantado; en el grado académico de las y los profesores; en los puestos laborales del personal docente, administrativo y de servicios; y en los distintos cargos de dirección.

La muestra ha sido extraída de dos centros públicos, como son la Universidad Autónoma de Nicaragua (UNAN) y la Universidad Nacional de Ingeniería (UNI), y dos privados de servicio público financiados por el Estado: la Universidad Centroamericana (UCA) y la Universidad Politécnica (UPOLI); todos ellos pertenecientes al Consejo Nacional de Universidades (CNU).

El estudio ha sido elaborado mediante revisión de fuentes documentales secundarias y recabando información estadística procedente de los registros de diferentes instancias de dichas universidades, las cuales se mencionan a continuación: en la UCA, la Dirección de Registro y Control Académico y la Dirección de Recursos Humanos; en la UNI, la Dirección de Registro; en la UPOLI, la División de Recursos Humanos y la Dirección de Educación Continua. Los datos de la UNAN fueron obtenidos de la publicación estadística La UNAN en Cifras 1998.

\section{La equidad en el acceso de varones y mujeres a la educación superior}

Desde los años setenta viene produciéndose en nuestro país, al igual que en el resto de la región y la subregión, una incorporación creciente de las mujeres a los distintos niveles del sistema educativo,

${ }^{2}$ La ponencia presentada por la CIEG en el Encuentro de Chile el pasado año, puede verse en Montecino y Obach, 1998. 
de tal modo que en el ciclo de primaria éstas constituyen hoy día la mitad de la matrícula (Valdés y Gomáriz, 1997:57). En secundaria, el porcentaje femenino de participación es ligeramente superior al masculino $(53.2 \%)$, más acorde con el porcentaje poblacional (Valdés y Gomáriz, 1997: 62). No obstante, la cobertura del acceso de las mujeres a estos niveles educativos es aún baja, ya que la tasas de matriculación femenina en primaria y secundaria son del 80.2 por ciento y 52.6 por ciento, respectivamente (PNUD 1999:32).

Si se toma en cuenta la distribución por sexo de la población que ha accedido a algún tipo de educación superior, se observa que también las distancias entre varones y mujeres han disminuído progresivamente. Así, en 1992, de un 4.2 por ciento de la población con formación universitaria, el 3.6 por ciento era femenino y el 4.9 por ciento masculino, correspondiéndose con el 44.6 por ciento de mujeres y el 55.4 por ciento de varones, del total de este grupo poblacional (Valdés y Gomáriz, 1997:54). Según datos más recientes, estas disparidades son aún menores en 1995 , con el 48 por ciento de mujeres y el 52 por ciento de varones que han adquirido instrucción universitaria (INEC, 1995:123). Por otro lado, la participación femenina en la escolarización terciaria ha tenido un crecimiento del 24 por ciento en los últimos diez años (PNUD 1999:32).

Asimismo, en tres décadas se ha producido una paulatina tendencia al equilibrio en la matrícula de la enseñanza superior, de tal modo que en 1975 la inscripción femenina alcanzaba un tercio del total; en 1980 superaba el 40 por ciento; en 1985 llegó al 56 por ciento, debido a la movilización militar de los varones por la guerra; y en 1992 era el 51.7 por ciento (Valdés y Gomáriz, 1997:64).

En los años 1995-96, un informe estadístico del Consejo Nacional de Universidades acerca de la matriculación estudiantil de los centros representados en esta instancia, arroja que la presencia de mujeres es de un 50 por ciento. Esta proporción se mantiene en nuestro muestra (Gráfico № 1), correspondiente a los años 1998-1999, en la que se reporta el 50 por ciento de varones $(11,881)$ y el 50 por ciento de mujeres $(11,808)$, de un total de 23,689 estudiantes. 


\section{Gráfico № 1}

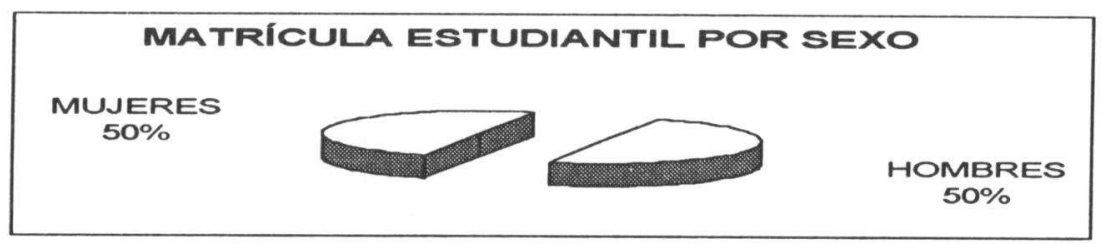

Fuentes: Dirección de Registro y Control Académico 1999, UCA.

La UNAN en Cifras 1998.

Dirección de Registro 1998, UNI.

Dirección de Educación Continua 1998, UPOLI.

Aun cuando inicialmente no se observan diferencias en el acceso a la educación superior, sí se perciben ciertas disparidades en la distribución de la matrícula por universidades y áreas de estudio. Y es que las relaciones de poder de género, no sólo se manifiestan en un desigual ingreso en la educación formal, sino que en el conjunto de los procesos de enseñanza-aprendizaje, a lo interno de la guardería, escuela, instituto y universidad, se encuentran activados diversos mecanismos sexistas, que moldean de forma diferenciada los intereses, las motivaciones, las personalidades femenina y masculina en general, repercutiendo en la configuración de las expectativas académicas y profesionales del alumnado.

\section{El sexismo en la labor educativa}

Investigaciones realizadas en otros países (Subirats, 1994), muestran cómo existen conductas rutinarias en la labor educativa, a menudo inconscientes y sutiles, que inferiorizan la vida femenina. Estos comportamientos son menos visibles que los institucionalizadas formalmente -por lo que se precisan instrumentos más elaborados para detectarlos- e influyen negativamente en la construcción de las identidades y la autoestima de género de las estudiantes mujeres.

En lo que concierne a la relación profesorado-estudiantes, dichas pautas de acción pueden ser identificadas, por ejemplo, en un diferente tipo de lenguaje en las interpelaciones docentes dirigidas al alumnado en función de su sexo. El sexismo profesoral, se puede apreciar asimismo en otros aspectos, tales como en el desigual grado de atención e interés prestado a los alumnos y alumnas, así como en la asignación asimétrica de responsabilidades, en las evaluaciones diferenciadas por sexo y en el chantaje sexual a las estudiantes. Las relaciones de poder genérico también se ponen de manifiesto en las interacciones entre las y los estudiantes: en el uso diferenciado de los 
espacios académicos y recreativos, en la interacción verbal pública, en la posibilidad o no de expresar afecto o agresividad, entre otras.

En relación estrecha con lo comentado en el apartado sobre el conocimiento científico, cabe señalar de manera más precisa, que el conocimiento seleccionado a través de la bibliografía y los textos didácticos oficiales de los diversos niveles educativos, responden, igualmente, a valores y concepciones del mundo androcéntricos y discriminatorios. En términos generales, en el discurso de los sistemas de pensamiento predominantes, reflejado en los materiales pedagógicos de las distintas disciplinas, o bien se silencian, o bien se menosprecian las capacidades femeninas, al simplificar y trivializar las experiencias históricas y los protagonismos individuales y colectivos de las mujeres.

En la tradición teórica e investigativa hegemónica transmitida en los textos de estudio, la devaluación de lo femenino responde comúnmente a estereotipos de género, en los cuales las mujeres aparecen asociadas a imágenes o conceptos maternales y domésticos, subvalorados socialmente; o también, a diversos modelos de erotismo. En cualquiera de las variantes posibles de estos estereotipos de género, las mujeres son caracterizadas como humana e intelectualmente reducidas, respecto a la simbología y tematización de la vida masculina, la cual es representada como un universal paradigma de conducta de la especie (Castillo, 1994).

Este conjunto de habituales situaciones educativas sexistas, incide negativamente en la formación de la subjetividad de las mujeres. Es decir, la discriminación de las mismas erosiona su autovaloración personal y, además, restringe sus ambiciones y proyectos vitales y profesionales, a las posibilidades y a los espacios que una sociedad sexista les atribuye como los apropiados y suficientes para ellas. Ello se suele expresar entre las estudiantes en el momento de seleccionar el tipo de carreras que desean cursar, temiendo elegir aquellas que socialmente están asignadas a los varones, pues les requerirán superar obstáculos propios de un mundo académico y profesional muy masculinizado. Por su parte, los varones rechazarán carreras netamente feminizadas, valorándolas como inadecuadas para su género. Esto se pone de manifiesto en el presente estudio, al distribuir la matrícula de la muestra estudiantil por casas de estudios (Gráfico № 2). Cabe precisar que metodológicamente denominamos paritarios a los valores que siendo femeninos o masculinos oscilen entre el 40 por ciento y el 60 por ciento. Consecuen-temente, serán consideradas masculinas y femeninas aquellas proporciones de varones y mujeres, respectivamente, que sobrepasen los 60 puntos. 
La UCA y la UPOLI son centros femeninos por su inscripción estudiantil, ya que ambos reportan un 61 por ciento de estudiantes mujeres y un 39 por ciento de varones, acorde con un predominio de áreas académicas asociadas a sectores laborales de servicios, habitualmente atribuidos a las mujeres. La UNAN es paritaria, con el 45 por ciento de inscripción masculina $(5,160)$ y el 55 por ciento

\section{Gráfico № 2}

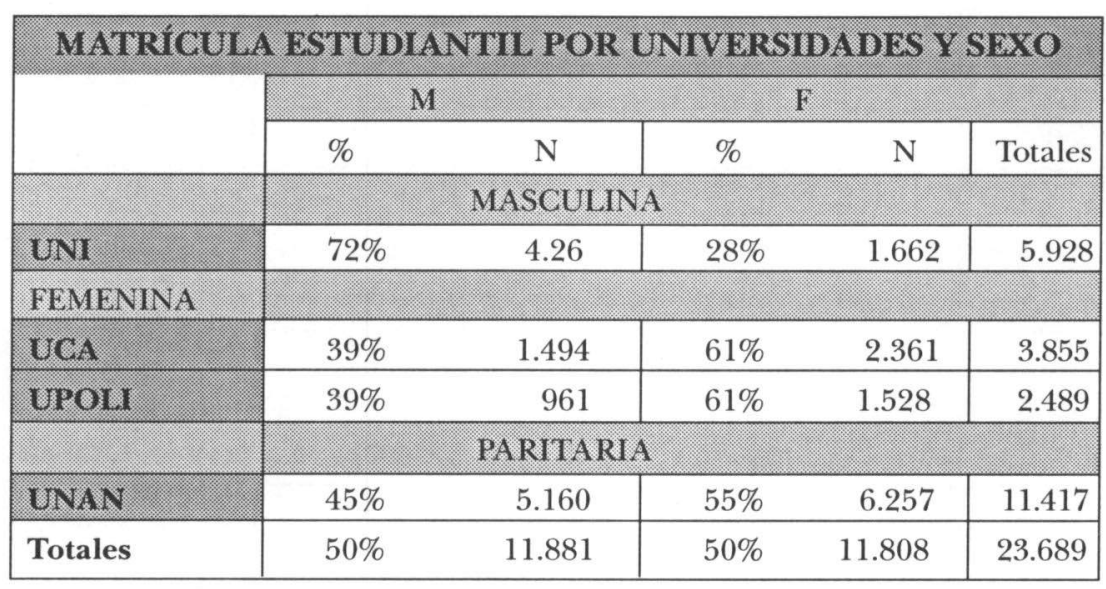

Fuentes: Dirección de Registro y Control Académico 1999, UCA.

La UNAN en Cifras 1998.

Dirección de Registro 1998, UNI.

Dirección de Educación Continua 1998, UPOLI.

femenina $(6,257)$, lo que se explica por la combinación de carreras tanto de tradición femenina como masculina, tales como las de Educación/Humanidades y las de Ciencias Exactas, respectivamente. La UNI es un centro fuertemente masculino, ya que de cada cien estudiantes 72 son varones $(4,266)$, de un total de 5,928 matriculados/as. Esta composición está en correspondencia con las carreras de ingeniería que se ofrecen en esta casa de estudios, más vinculadas a la producción industrial, es decir, a ámbitos laborales de tradición masculina. La distribución de estudiantes por áreas académicas, pone aún más claramente en evidencia las diferencias en la preferencia de estudio. En el gráfico № 3 se observa que son femeninas las áreas de Ciencias de la Comunicación, Ciencias de la Educación/Sociales/Humanidades y Ciencias Médicas y de la Salud, con una matrícula de mujeres superior al 60 por ciento, en concordancia con la situación latinoamericana en general (Valdés y Gomáriz, 1995:109). 


\section{Gráfico № 3}

\begin{tabular}{|c|c|c|c|c|c|}
\hline & POI & AREA & S DE E & TUDIC & Y SEXO \\
\hline & \multicolumn{2}{|c|}{$\mathrm{M}^{2}$} & \multicolumn{2}{|c|}{$F$} & \multirow[b]{2}{*}{ Totales } \\
\hline & $\%$ & $\mathrm{~N}$ & $\%$ & $\mathrm{~N}$ & \\
\hline & \multicolumn{4}{|c|}{ FEMENINAS } & \\
\hline $\begin{array}{l}\text { Ciencias de la Educación/Sociales } \\
\text { y Humanidades }\end{array}$ & $32 \%$ & 1.540 & $68 \%$ & 3.264 & 4.804 \\
\hline Ciencias Médicas y de la Salud & $33 \%$ & 791 & $67 \%$ & 1.639 & 2.430 \\
\hline \multirow[t]{2}{*}{ Ciencias de la Comunicación } & $37 \%$ & 107 & $63 \%$ & 183 & 290 \\
\hline & \multicolumn{5}{|c|}{ MASCULINAS } \\
\hline \multirow[t]{2}{*}{ Ingeniería } & $75 \%$ & 3.811 & $25 \%$ & 1.281 & 5.092 \\
\hline & \multicolumn{5}{|c|}{ PARTTARIAS } \\
\hline Arquitectura & $50 \%$ & 225 & $50 \%$ & 222 & 447 \\
\hline $\begin{array}{l}\text { Ciencias Administrativas, } \\
\text { Económicas y Comerciales }\end{array}$ & $52 \%$ & 2.967 & $48 \%$ & 2.731 & 5.698 \\
\hline $\begin{array}{l}\text { Ciencias Exactas/ Informática }{ }^{3} \\
\text { Ciencias Agropecuarias/ }\end{array}$ & $54 \%$ & 1.336 & $46 \%$ & 1.155 & 2.491 \\
\hline Medioambientales $^{4}$ & $53 \%$ & 220 & $47 \%$ & 196 & 416 \\
\hline Ciencias Jurídicas & $43 \%$ & 761 & $57 \%$ & 1.009 & 1.770 \\
\hline Sin especificar ${ }^{5}$ & $49 \%$ & 123 & $51 \%$ & 128 & 251 \\
\hline Totales & $50 \%$ & 11.881 & $50 \%$ & 11.808 & 23.689 \\
\hline
\end{tabular}

Fuentes: Dirección de Registro y Control Académico 1999, UCA.

La UNAN en Cifras 1998.

Dirección de Registro 1998, UNI.

Dirección de Educación Continua 1998, UPOLI.

${ }^{3}$ En este área académica de Ciencias e Informática, se incluyen todas las carreras de la Facultad de Ciencias de la UNAN, es decir, Estadística, Química, Física, Electrónica, Matemática, Biología, Geología y Computación, así como la carrera de la Facultad de Ciencias y Sistemas de la UNI. De la UPOLI, se seleccionan para este área las carreras de Estadísticas Económicas, Ciencias de la Computación e Ingeniería en Sistemas.

${ }^{4}$ Se incluyen aquí las carreras de la Facultad de Ciencias Agropecuarias de la UCA: Zootecnia, Ecología, Ecología y Desarrollo, y Nutrición, así como las carreras de Ingeniería en Sistemas de Producción Agropecuaria 
Conviene puntualizar que si bien este último área es de carácter femenino, una distribución por carreras muestra que Medicina es paritaria, con el 53 por ciento de mujeres y el 47 por ciento de varones, pero las Enfermerías continúan convocando mayoritariamente a las mujeres, con más del 80 por ciento. En el mismo sentido, la carrera de Pedagogía también muestra una representación femenina superior al 80 por ciento y en la de Educación Preescolar de la UNAN la proporción de alumnas es del 99 por ciento. Por el contrario, las carreras de Ingeniería persisten como un medio masculino por antonomasia, con una inscripción de mujeres de menos de un tercio del total.

Con todo, las demás áreas disciplinarias son paritarias, por lo que es posible que Nicaragua continúe siendo actualmente, el país de la región latinoamericana que mayores oportunidades proporciona a las mujeres en la formación superior, al poseer solamente un área de estudio masculina (Valdés y Gomáriz, 1995:110).

\section{Las asimetrías genéricas en la organización universitaria}

La estructura organizativa de la universidad, manifiesta igualmente una jerarquización basada en la desigualdad de género. Estas asimetrías se observan en la institución, tomando en cuenta las diferentes posiciones de varones y mujeres en los diversos sectores laborales, así como en los distintos niveles de formación profesional y de dirección del sistema universitario.

\section{Personal subalterno de Administración y Servicios}

El sector del personal subalterno de administración y servicios de las cuatro universidades en estudio, tiene una composición mayoritariamente femenina, ya que de un total de 1,067 personas reportadas, el 61 por ciento (652) son mujeres y el 39 por ciento (415) varones.

e Ingeniería en Calidad Ambiental, de la misma universidad. La carrera de Administración Agropecuaria de la UPOLI se incluye igualmente en este área de Ciencias Agropecuarias.

${ }^{5}$ Se trata de las carreras de Diseño Industrial (33 varones y 68 mujeres) y Diseño Gráfico (90 varones y 60 mujeres), de la UPOLI. 
Como se aprecia en el gráfico № 4 , se produce una gran segmentación en el apoyo administrativo, con una presencia masiva de mujeres, esto es, el 83 por ciento (360) de un total de 436 personas. Las actividades de servicios aparecen globalmente como paritarias, el 54 por ciento (339) de varones y el 46 por ciento (292) de mujeres. Esta paridad se debe a que los oficios de carpintería, albañilería, electricidad, vigilancia y transporte, habitualmente mejor remunerados que los del servicio de limpieza, son exclusivos de los hombres. Por el contrario, la participación femenina aparece fundamentalmente en los puestos de afanadora y cocinera, aunque especialmente los primeros, no son exclusivos de las mujeres.

\section{Gráfico № 4}

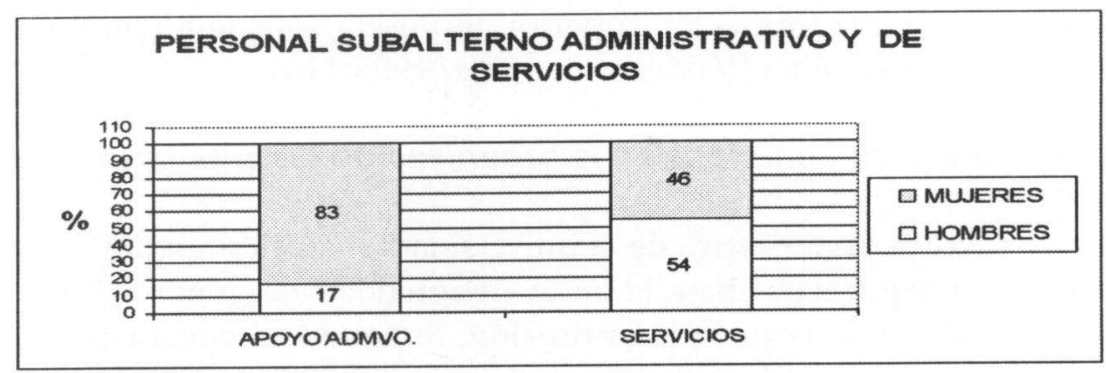

Fuentes: Dirección de Recursos Humanos 1999, UCA.

La UNAN en Cifras 1998.

Dirección de Registro 1998, UNI.

División de Recursos Humanos 1999, UPOLI.

La información muestra la existencia de una fuerte división de los oficios, asociada a los roles tradicionales de varones y mujeres. Esto pone al descubierto una pobre sensibilidad y escasos esfuerzos para promover, en este nivel de la comunidad universitaria, una diversificación laboral que contribuya a la disminución de las asimetrías socio-económicas basadas en las diferencias sexo-genéricas.

\section{Áreas académicas del profesorado}

El número de profesores/as de pregrado de esta muestra, asciende a 1,273. De ellos, $775(61 \%)$ son varones y $498(39 \%)$ mujeres (Gráfico № 5). 


\section{Gráfico № 5}

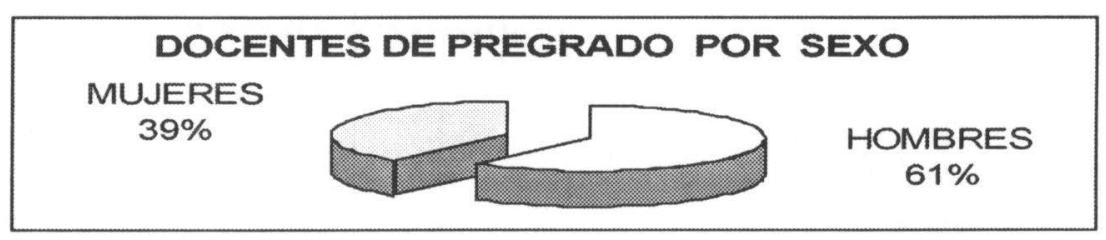

Fuentes: Dirección de Recursos Humanos 1999, UCA.

La UNAN en Cifras 1998.

Dirección de Registro 1998, UNI.

División de Recursos Humanos 1999, UPOLI.

Las proporciones femeninas ascienden 3 puntos respecto a las encontradas en estudios realizados en 1992-1993 (Valdés y Gomáriz, 1997:66). Esto podría estar indicando un incremento paulatino, aunque lento, de la presencia de mujeres en el plantel docente. No obstante, también en ese mismo estudio se destaca que las mujeres participan masivamente en los ciclos de preescolar y primaria, alcanzando un 99 por ciento y un 85 por ciento, respectivamente, pero su representación disminuye de modo significativo en secundaria, con un 57 por ciento; y más aún en la universidad, con un 36 por ciento.

Estos sesgos de género están asociados a una distribución sexogenérica del trabajo, que asigna a las mujeres los estratos más bajos de cualificación profesional en la enseñanza, como una suerte de prolongación de sus roles maternales y domésticos en el ámbito familiar. Por el contrario, cuando la profesión educativa exige una mayor formación y, además, se encuentra mejor remunerada, socialmente se estima que es una tarea más específicamente masculina, por lo que la proporción entre varones y mujeres tiende a revertirse.

Esto ocurre más claramente en los niveles de mayor prestigio y estatus socio-económico como es el universitario, y en especial en las áreas académicas más valoradas de los estudios superiores. De ahí que el porcentaje de mujeres docentes universitarias (39\%), no se corresponda con las proporciones equitativas de matriculación femenina estudiantil, ni con el igualmente alto grado de paridad encontrado en las áreas de estudio según el sexo de los estudiantes. 


\section{Gráfico № 6}

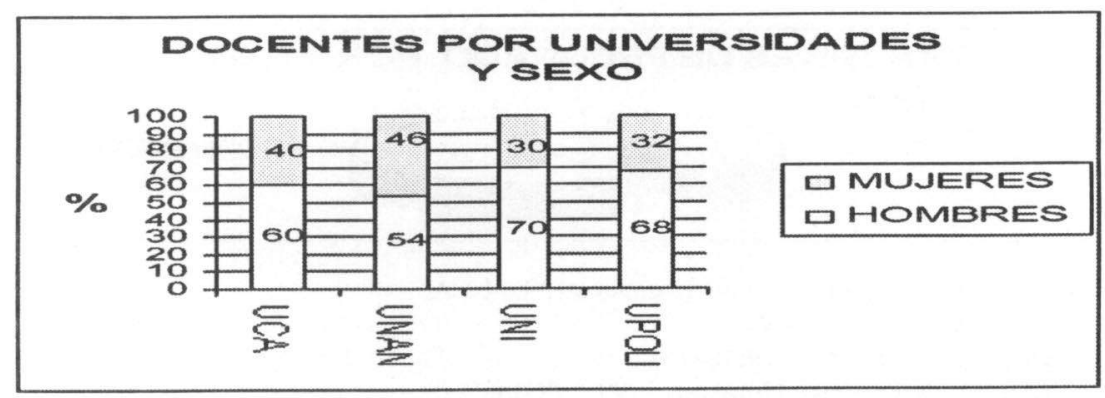

Fuentes: Dirección de Recursos Humanos 1999, UCA.

La UNAN en Cifras 1998.

Dirección de Registro 1998, UNI.

División de Recursos Humanos 1999, UPOLI.

Todas las universidades tienen en su planta docente más profesores hombres que mujeres (Gráfico 6), aunque la UNAN y la UCA presentan proporciones paritarias, ya que de cada cien docentes 54 son varones (284) y 46 mujeres (244) en el primer centro, y en el segundo 60 (175) y 40 (114), respectivamente. La UNI posee un 70 por ciento (198) de docentes hombres, de un total de 282 , en correspondencia con una matrícula estudiantil de ingeniería mayoritariamente masculina. Le sigue la UPOLI, con un 68 por ciento (118) de profesores varones, de un total de 174 .

En la distribución del profesorado por áreas académicas, no aparece ningún área disciplinaria universitaria en la que las mujeres docentes estén representadas por encima del 60 por ciento (Gráfico № 7). Únicamente son paritarias el área de Ciencias de la Educación/Sociales/Humanidades y Ciencias Médicas y de la Salud, aunque la carrera de Medicina arroja un 70 por ciento de docentes varones, que es equilibrado con un 88 por ciento de profesoras de la carrera de Enfermería.

La participación masculina predominante entre los profesionales de la enseñanza superior, se observa incluso en carreras femeninas en cuanto a matriculación estudiantil. Este es el caso de las Ciencias de la Comunicación, que reporta un 71 por ciento de profesores varones, a pesar de que existe un 63 por ciento de mujeres cursando estos estudios. Nótese asimismo, que las Ciencias Administrativas, Económicas y Comerciales, pese a contar con una inscripción estudiantil equitativa, destaca por la masculinización 
de su profesorado, que alcanza un 75 por ciento del total de docentes y se sitúa 2 puntos por encima de las carreras de ingeniería.

\section{Gráfico № 7}

\begin{tabular}{|c|c|c|c|c|c|}
\hline \multicolumn{6}{|c|}{ DOCENTES POR AREAS DE ESTUDIO Y SEXO } \\
\hline & \multicolumn{2}{|c|}{$M$} & \multicolumn{2}{|c|}{$\mathrm{F}$} & \\
\hline & $\%$ & $\mathrm{~N}$ & $\%$ & $\mathrm{~N}$ & Totales \\
\hline & \multicolumn{4}{|c|}{ FEMENINAS } & \\
\hline & \multirow{2}{*}{\multicolumn{4}{|c|}{ MASCULINAS }} & \\
\hline & & & & & \\
\hline $\begin{array}{l}\text { Ciencias Admvas., } \\
\text { Económicas y Comerciales }\end{array}$ & $75 \%$ & 149 & $25 \%$ & 50 & 199 \\
\hline Ingeniería & $73 \%$ & 133 & $27 \%$ & 48 & 181 \\
\hline Comunicación & $71 \%$ & 10 & $29 \%$ & 4 & 14 \\
\hline Arquitectura & $69 \%$ & 22 & $31 \%$ & 10 & 32 \\
\hline Ciencias Jurídicas & $68 \%$ & 63 & $32 \%$ & 29 & 92 \\
\hline C.c. Exactas/Informática & $62 \%$ & 118 & $38 \%$ & 72 & 190 \\
\hline \multirow[t]{2}{*}{$\begin{array}{l}\text { Ciencias Agropecuarias y } \\
\text { Medioambientales }\end{array}$} & $62 \%$ & 13 & $38 \%$ & 8 & 21 \\
\hline & \multicolumn{4}{|c|}{ PARITARIAS } & \\
\hline $\begin{array}{l}\text { Ciencias de la Educación/ } \\
\text { Sociales y Humanidades }\end{array}$ & $43 \%$ & 99 & $57 \%$ & 129 & 228 \\
\hline Ciencias Médicas y de la Salud & $55 \%$ & 120 & $45 \%$ & 99 & 219 \\
\hline Sin especificar ${ }^{6}$ & $57 \%$ & 29 & $43 \%$ & 23 & 52 \\
\hline Totales $^{7}$ & $62 \%$ & 756 & $38 \%$ & 472 & 1.228 \\
\hline
\end{tabular}

Fuentes: Dirección de Recursos Humanos 1999, UCA.

La UNAN en Cifras 1998.

Dirección de Registro 1998, UNI.

División de Recursos Humanos 1999, UPOLI.

${ }^{6}$ Se trata de 17 profesores y 15 profesoras de Estudios Generales (UCA), más 12 varones y 8 mujeres docentes de las carreras de Diseño (UPOLI).

${ }^{7}$ Estos porcentajes totales del profesorado varían en un punto respecto a los anteriores, debido a que en la UCA no concuerda el total de docentes en general, con el total reportado en la distribución de docentes por áreas de estudio y grados académicos. En el primer caso se reportan $175(60 \%)$ varones y $114(40 \%)$ mujeres, que incluye a profesores de tiempo completo y horarios; y en el segundo $156(64 \%)$ hombres y $88(36 \%)$ mujeres, en el que sólo se contabilizan los profesores horarios. Ver análisis del Gráfico № 6 . 
Estos datos podrían estar indicando una revalorización laboral de estas áreas académicas, asociada a un control masculino de sectores sociales y profesionales en auge desde el cambio de sistema económico y político en 1990, tales como la empresa privada y los diversos medios de comunicación social.

En general, este contexto asimétrico sugiere la existencia de algún tipo de favoritismo en la contratación masculina, no acorde con la formación femenina en estudios superiores. Se trataría de obstáculos informales, como son las conductas de ocultación o distorsión, basadas en los estereotipos sexistas mencionados anterior-mente, acerca de las cualidades intelectuales y profesionales de las mujeres. Estos impedimentos están presentes, consciente o inconscientemente, en el momento de la contratación, en especial cuando la normativa al respecto no está suficientemente explicitada o se halla supeditada a los contactos e influencias que puedan tener las y los aspirantes dentro de la universidad (Acker, 1997).

Además, existen también trabas estructurales relacionadas con la exigencia de dedicación exclusiva y de rendimiento que conlleva la labor educativa, sobre todo para las profesionales mujeres de la enseñanza universitaria. Ello es difícilmente compatible con la gran inversión de tiempo, energías y emociones que exigen las responsa-bilidades domésticas y familiares, todavía hoy mayoritariamente en manos femeninas; dificultades que aumentan con la competitividad profesional, asociada a la creciente inestabilidad en el mercado laboral académico de los centros de educación superior.

\section{Grado académico de docentes}

Las supremacía masculina se ve reforzada por la existencia de una mayor proporción de varones con formación posgraduada, aunque existen diferencias relevantes entre titulaciones (Gráfico № 8). Así, de un total de 142 docentes con el título de Doctorado, sólo el 25 por ciento (35) son mujeres. El título de Maestría, sin embargo, tiene un porcentaje más igualitario, es decir, las mujeres graduadas en este nivel son el 43 por ciento (117) de un total de 274. En los grados de especialistas y licenciados/as, se observa igualmente una composición paritaria, esto es, el 48 por ciento (50) y el 41 por ciento (241) de mujeres, respectivamente. 


\section{Gráfico № 8}

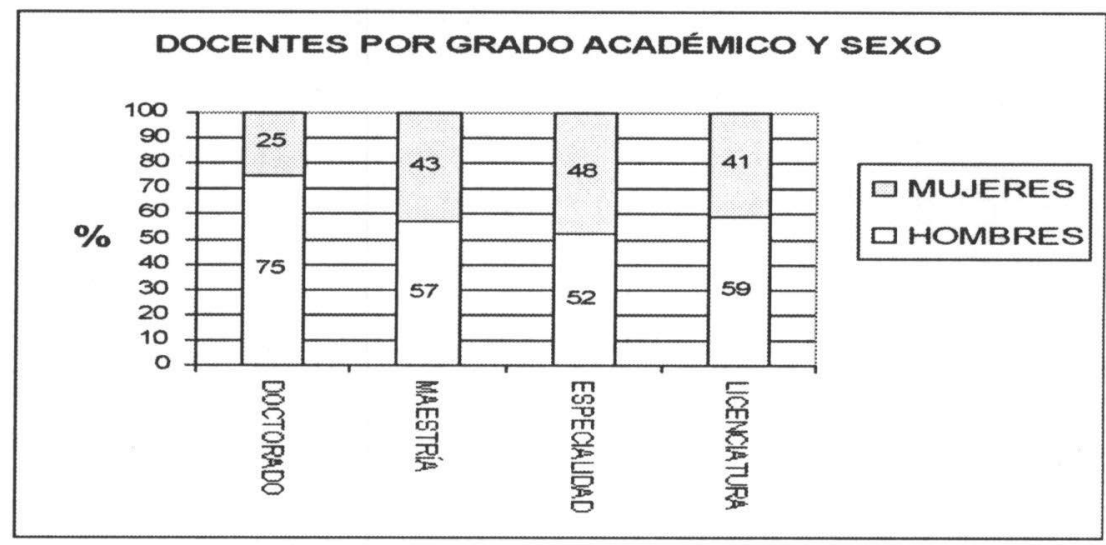

Fuentes: Dirección de Recursos Humanos 1999, UCA.

La UNAN en Cifras 1998.

Dirección de Registro 1998, UNI.

División de Recursos Humanos 1999, UPOLI.

En los dos gráficos siguientes, se aprecian asimismo contrastes significativos entre universidades. En términos de grados doctorales (Gráfico 9), la UPOLI ${ }^{8}$ presenta la mayor segmentación, ya que solamente cuenta con doctores varones (3). Le sigue la UCA en predominio masculino doctoral, pues del total de sus docentes con este título (10), solamente son mujeres el 20 por ciento (2). La UNAN posee el 24 por ciento de mujeres (28), frente al 76 por ciento de varones (89). La UNI muestra los porcentajes más ponderados en este nivel de graduación: el 58 por ciento de sus doctores son varones (7) y el 42 por ciento son mujeres (5), lo cual es un dato a subrayar, ya que se trata de una universidad básicamente masculina tanto en la composición por sexo de su alumnado (72\%), como de su profesorado (70\%).

En cuanto al grado de Maestría (Gráfico 10), destaca la UNAN mostrando la situación más equitativa, ya que de 136 docentes con esta graduación, la mitad son mujeres (68). La UPOLI sobresale con

${ }^{8}$ Se proporcionan solamente los grados académicos de los/as docentes de tiempo completo de la UPOLI (26 varones y 27 mujeres). Este número no coincide con el total de docentes contabilizado en el análisis de las áreas de estudio (118 hombres y 56 mujeres). Ver análisis del Gráfico № 6 . 


\section{Gráfico № 9}

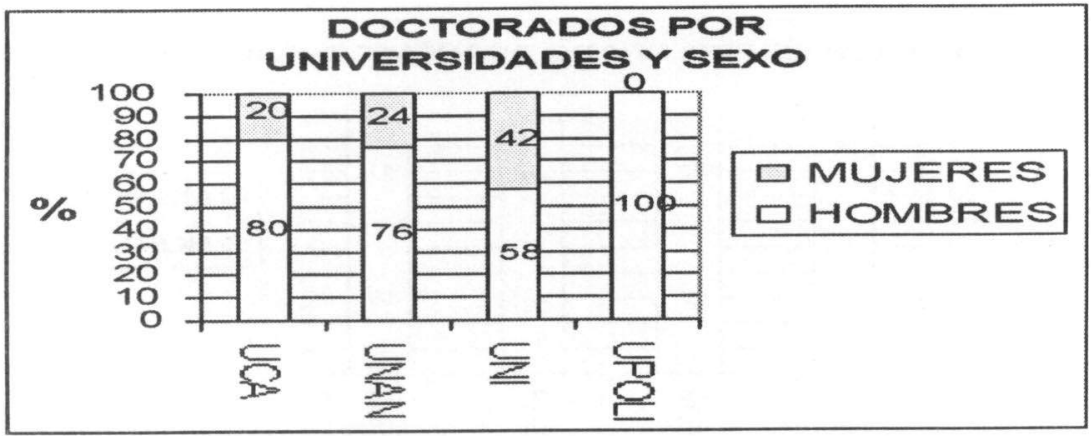

Fuentes: Dirección de Recursos Humanos 1999, UCA.

La UNAN en Cifras 1998.

Dirección de Registro 1998, UNI.

División de Recursos Humanos 1999, UPOLI.

el 69 por ciento de titulación femenina (9) y el 31 por ciento masculina (4). En sentido contrario se distingue la UNI, que cuenta con un 29 por ciento de mujeres (18) y un 71 por ciento de varones (45). En la misma línea de preponderancia masculina, le sigue la UCA, con un 35 por ciento de mujeres (22) y un 65 por ciento de hombres (40).

\section{Gráfico № 10}

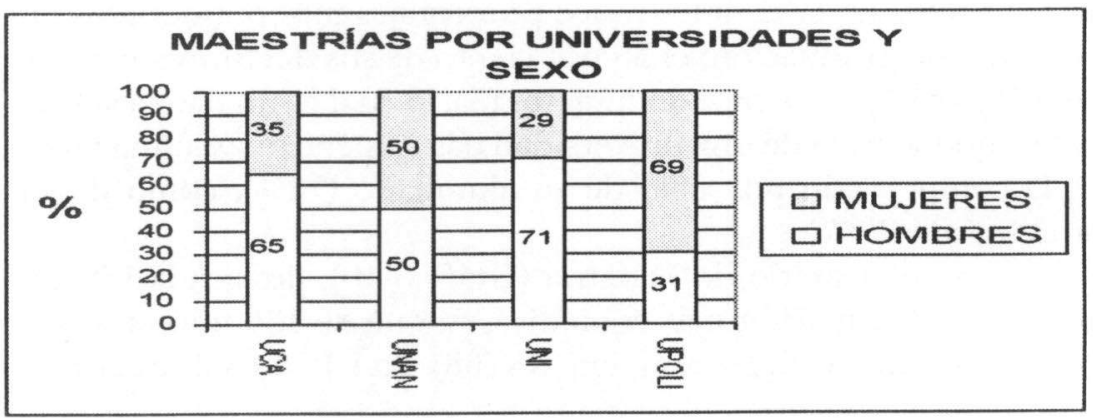

Fuentes: Dirección de Recursos Humanos 1999, UCA.

La UNAN en Cifras 1998.

Dirección de Registro 1998, UNI.

División de Recursos Humanos 1999, UPOLI. 
Esta información apunta en general a la existencia de mayores dificultades para las mujeres profesionales de la enseñanza, al momento de acceder a los estudios postgraduados de más alto nivel. Dicha formación académica exige mucho esfuerzo y tiempo de dedicación y, además, a menudo se requiere salir del país, para todo lo cual las responsabilidades familiares suelen representar fuertes obstáculos, que no suelen tomarse en cuenta en las políticas de becas o en las oportunidades de formación. Asimismo, de modo similar a lo que sucede en las contrataciones del profesorado, pueden existir preferencias sexistas en las personas responsables de la cualificación de la planta docente.

\section{Puestos directivos}

En los niveles más altos de decisión (Gráfico № 11), las asimetrías en la participación de varones y mujeres se incrementan considerablemente respecto al estamento docente, ya que del total de personas que ocupan los puestos directivos estudiados (107), el 70 por ciento (75) son varones y el 30 por ciento mujeres (32).

\section{Gráfico № 11}

DIRECTIVOS POR SEXO

MUJERES

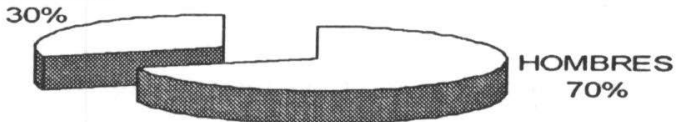

Fuentes: Dirección de Recursos Humanos 1999, UCA.

La UNAN en Cifras 1998.

Dirección de Registro 1998, UNI.

División de Recursos Humanos 1999, UPOLI.

Existe un predominio masculino en todas las instancias de dirección (Gráfico 12), salvo en el cargo de Vicerrectoría, que muestra un 55 por ciento (6) de participación femenina.

Así, en Rectorías y Secretarías Generales ${ }^{9}$ se da una presencia

${ }^{9}$ La UPOLI y la UCA no cuentan con Secretarías Generales. 


\section{Gráfico № 12}

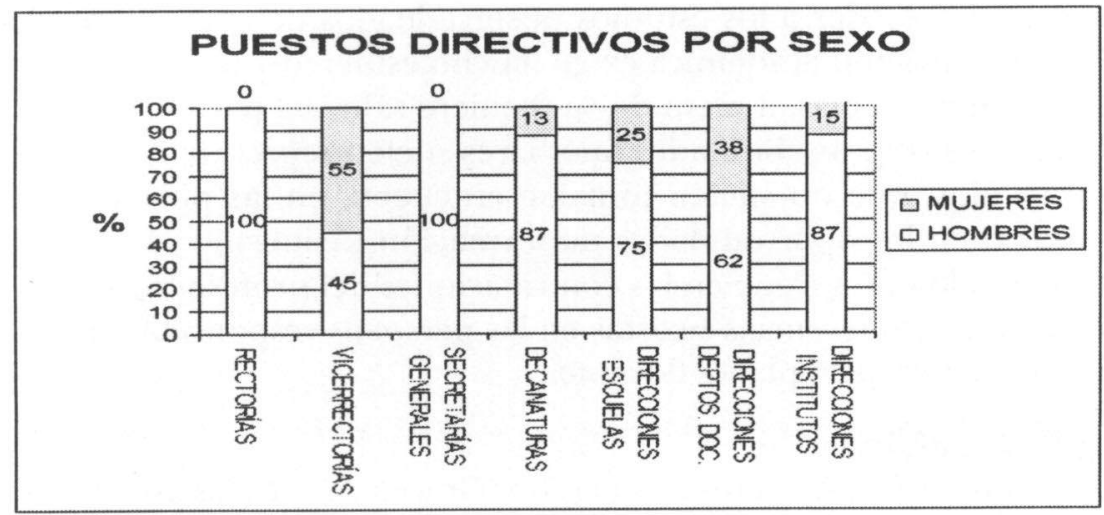

Fuentes: Dirección de Recursos Humanos 1999, UCA.

La UNAN en Cifras 1998.

Dirección de Registro 1998, UNI.

División de Recursos Humanos 1999, UPOLI.

exclusiva de varones (100\%). Le siguen en orden de importancia en cuanto a hegemonía masculina, las Direcciones de Institutos y Centros adscritos (13) y los cargos de Decanaturas (14), que en ambos casos concentran una presencia de varones del 87\%. Las Direcciones de Escuela tiene el 75 por ciento de representación masculina (3). Las Direcciones de los Departamentos Docentes ${ }^{10}$ son las instancias inferiores de esta escala, y son asimismo masculinas, aunque reportan el porcentaje más alto de representación femenina después de las Vicerrectorías, esto es, el 38 por ciento (21), frente al 62 por ciento masculino (34).

En el caso de las Vicerrectorías, la UNI y la UPOLI son los centros universitarios que determinan el carácter equitativo global de este puesto de dirección, ya que poseen una presencia femenina del 75 por ciento (3) y 100 por ciento (1), respectivamente, frente al 67 por ciento (2) de vicerrectores varones en cada una de las universidades restantes, es decir, la UCA y la UNAN.

En la UNAN y en la UNI, las Decanaturas están a cargo exclusivo de varones (4 y 6 decanos, respectivamente). Estas cifras son menos

${ }^{10}$ No se cuenta con datos de las Direcciones de Departamentos Docentes de la UNAN. 
extrañas en lo que respecta a la UNI, por ser una universidad mayoritariamente masculina en términos de matricula estudiantil y de profesorado, pero resultan más sorprendentes en la UNAN, que tiene en este sentido, un carácter paritario, es decir, el 55 por ciento y el 46 por ciento de mujeres, estudiantes y docentes, respectivamente. La UCA es el centro universitario que cuenta con mayor participación femenina en este nivel de dirección, si bien con un tercio del total de decanos (2 de 6). La UPOLI únicamente posee un 25 por ciento (1) de mujeres como Directoras de Escuela ${ }^{11}$.

En los datos sobre Direcciones de Departamentos Docentes reportados por las tres universidades, se encuentra que la UNI concentra la mayor representación masculina, con el 68 por ciento de varones (26) y el 32 por ciento de mujeres (12). En la UPOLI, pese a reportar un número más alto de hombres que de mujeres en esta instancia, se observa una proporción paritaria, con el 57 por ciento (4) masculino y el 43 por ciento (3) femenino. Contrariamente, la UCA muestra una composición numérica que favorece a las mujeres y con un porcentaje paritario, es decir, el 40 por ciento (4) es masculino y el 60 por ciento femenino (6).

Llama poderosamente la atención, la proporción mayoritariamente masculina de las Direcciones de Institutos y Centros adscritos $(87 \%)^{12}$, destacándose al respecto la UNAN y la UPOLI, con el 100 por ciento de directores hombres, en todos los centros autónomos reportados. La labor de dirección de estas instituciones adscritas, es significativa respecto al poder de género, ya que se dedican básicamente a la investigación, a la capacitación postgraduada y a proyectos sociales, gozando de un alto grado de autonomía en términos de capacidad de decisión y control financiero, además de amplias posibilidades de promoción profesional.

A la luz de estos datos, se advierte que la conducción de estas casas de estudios, vista en su conjunto, posee una fuerte brecha sexo-genérica, más acusada aún que la que se produce en el estamento profesoral. De manera similar a lo que ocurre en la escala docente de todo el sistema educativo, se aprecia que, a medida

${ }^{11} \mathrm{El}$ sistema de Escuelas rige únicamente en la UPOLI.

${ }^{12}$ Los Institutos y Centros adscritos reportados son: en la UCA, JUAN XXIII, CASC, Nitlapan e IHNCA; en la UNAN, CIRA, CIES, INIES, CIGEO, CISE Y CINET; en la UNI, IES; y en la UPOLI, IDEHU, IMLK, ICIDRI, CIELAC Y CASIPJ. 
que se asciende en la jerarquía de las universidades, la representación femenina tiende a disminuir significativamente, a excepción en este caso de las Vicerrectorías, que tienen una composición paritaria.

Los obstáculos sugeridos anteriormente, que explicarían la menor contratación y cualificación de mujeres docentes universitarias, es decir, aquellos relacionados con las prejuicios discriminatorios, así como con las responsabilidades afectivo-familiares femeninas, pueden ser igualmente válidos para dar cuenta de esta asimétrica organización de las universidades.

Cabe agregar la existencia de trabas solapadas, que las mujeres encuentran en círculos y redes de poder aplastantemente masculinos. A modo de ejemplo, pueden mencionarse las exigencias institucionalizadas informalmente, acerca del tipo de lenguaje y el tono de voz a utilizar en las reuniones, el requerimiento de una vestimenta femenina "apropiada" en un espacio masculinizado, así como la doble moral sexo-genérica, que controla y sanciona la reputación sexual, el "equilibrio" emocional y la atención a la familia, de manera más restrictiva en las mujeres que en los varones, etc. Todos los escollos mencionados, tanto internos como externos a la universidad, contribuyen a frenar la participación femenina en estas instancias directivas.

Finalmente, este sistema de organización viene a desempeñar un papel primordial de socialización genérica, consolidando las identidades femenina y masculina tradicionales, así como los estereotipos sexistas que atribuyen a los varones la capacidad de toma de decisiones, control y dirección y a las mujeres un rol secundario.

\section{Conclusiones}

En términos generales, esta investigación nos permite concluir que en la actualidad estamos ante un proceso creciente de equiparación cuantitativa de varones y mujeres en la matrícula estudiantil universitaria. No obstante, todavía persiste una sobrerrepresentación masculina en áreas de estudio relacionadas con las profesiones productivas y mejor remuneradas, como son las carreras de ingeniería, así como una concentración femenina en carreras ligadas a los sectores de servicios, tales como la de Pedagogía y la de Enfermería, de menor prestigio profesional y con salarios inferiores. 
Análogamente, estas circunstancias se expresan en el estamento del personal subalterno de administración y servicios, donde el apoyo administrativo se encuentra predominantemente en manos femeninas y los oficios de servicios más ligados a la producción son exclusivos de los varones. Asimismo, persiste una organización significativamente dispar en la planta de profesores de pregrado, con una mayor participación masculina en la totalidad de las áreas de estudio; brecha que se acentúa en la distribución por sexo de los grados académicos del profesorado, así como en las instancias de dirección universitaria tomadas en conjunto.

Tales fenómenos responden a una manifestación de la división sexo-genérica del trabajo en el ámbito de la educación superior, que está asociada a preconcepciones sexistas contra las mujeres y a otras prácticas y estructuras discriminatorias, internas y externas a la universidad, dando como resultado un sólido entretejido de relaciones de poder de género en el sistema universitario. Este orden jerarquizado se retroalimenta al actuar como modelo de socialización, dispuesto a ser introyectado y reproducido inadvertidamente por el conjunto de actores de la comunidad universitaria, reforzando así la percepción tradicional que varones y mujeres puedan tener de sus capacidades profesionales y expectativas vitales como género.

Ante esta situación, se precisan políticas nacionales orientadas a la eliminación de las desigualdades en el conjunto del sistema educativo, así como a una redistribución equitativa del mercado de trabajo y de las responsabilidades familiares entre varones y mujeres. La ausencia de ellas no es razón suficiente para que las máximas autoridades de la educación superior en particular y la comunidad universitaria en general, eludan enfrentarse a una revisión profunda de la realidad universitaria con vistas a democratizar las relaciones de género.

Ésta ha de implicar, entre otras acciones, la transformación de los planes de orientación vocacional del estudiantado y del currículo en su más amplio sentido. Asimismo, ha de requerir la elaboración de políticas y programas de investigación, contratación, capacitación y promoción, que fomenten de forma efectiva la equidad entre varones y mujeres, en todos los espacios y planos de la comunidad universitaria. De este modo, los centros de educación superior podrán contribuir más adecuadamente a una formación integral, mostrándose ante la sociedad nicaragüense como agentes de un desarrollo comprometido indisolublemente con la inteligencia crítica y con los valores de democracia y justicia social. 
Es importante reiterar, que este estudio constituye un intento inicial de develar algunas de las asimetrías de género presentes en el ámbito universitario, mediante la revisión de datos cuantitativos. No obstante, queda planteado el reto de una mayor profundización, que ponga al descubierto más nítidamente aquellas actitudes y prácticas "naturalizadas", que por su mayor sutileza requieren métodos de análisis cualitativos y cuantitativos más complejos. Una investigación de este tipo vendría a respaldar y complementar las alternativas de transformación de las estructuras formales de estas casas de estudios y, asimismo, permitiría diseñar propuestas a favor de la equidad de género en la vida cotidiana del salón de clases.

\section{Implicaciones para el trabajo social}

Las ciencias humanísticas en general y el Trabajo Social en particular, son fundamentalmente femeninas, tal como revela este estudio. Ello tiene implicaciones en el status de la profesión de Trabajo Social. Es vista como una profesión devaluada en un contexto donde el sistema neo-liberal y el orden androcéntrico, van de la mano para crear abismales desigualdades sociales.

Muchos son los factores que han incidido en esta desvalorización, y que merecen ser discutidos a profundidad. Sin embargo, es posible señalar algunos que de singular importancia: Desde su nacimiento, ha sido considerado como una extensión de los roles femeninos, lo que desmerece socialmente, puesto que se piensa que éstas tareas están en el orden de lo "natural". Se espera que el perfíl ideal de la Trabajadora Social, responda con los mandatos sociales asignados al Género femenino, tales como capacidad de establecer relaciones de empatía, tener sensibilidad al dolor ajeno etc.

Dirige su accionar en busca del bienestar de las mujeres y la niñez fundamentalmente, los cuales son sectores sociales subalternos, ya que no cumplen con las características del Arquetipo viril protagonista de la historia. (Moreno, 1994). Por tanto, todo aquellas acciones que se asocien a estos grupos, no tendrán la misma importancia, si fueran con hombres dedicados al espacio público, valorado socialmente.

Nuestra identidad de Género femenina y la identidad profesional, dan orígen a posiciones subordinadas a lo interno de las Instituciones. Como mujeres y como futuras profesionales de Trabajo Social, no se nos socializa a ser asertivas, a tomar decisiones importantes, a 
ser emprendedoras. Prevaleciendo casi siempre la pasividad y el miedo a los retos. Sin duda hay muchas Trabajadoras sociales que permanecen en el anonimato y sus iniciativas de proyectos son presentados por sus jefes varones, dejando de ser visibilizadas como "protagonistas" del desarrollo humano.

Existe en el imaginario colectivo del estudiantado, la idea que no deben invertir sus esfuerzos en el estudio de una profesión que no es bien remunerada, lo que incide en la baja matrícula a carreras como Trabajo Social. Esta situación crea retos para el Trabajo Social nicaragüense. A saber: Develar la relaciones de poder entre los Géneros en sus diferentes manifestaciones, evitar las prácticas sexistas en la enseñanza del Trabajo Social y asumir la perspectiva de Género, tanto en su aporte teórico como metodológico, en la enseñanza y en la práctica de la profesión.

\section{Bibliografía}

Acker, Sandra (1995). Género y Educación: Reflexiones sociológicas sobre mujeres, enseñanza y feminismo. Madrid, España: Narcea.

Bonder, Gloria (comp.) (1994). Igualdad de Oportunidades para la Mujer: Un desafío a la Educación Latinoamericana. Buenos Aires, Argentina: Ministerio de Cultura y Educación.

Brown, Harold I (1984). La nueva filosofía de la ciencia. Madrid, España: Tecnos.

Castillo, Vilma (1994). El Sexismo en la Escuela. En Irela Solórzano y Humberto Abaunza, Las reglas del juego: Limites y libertades de la juventud en Nicaragua. Managua, Nicaragua: Puntos de Encuentro.

Consejo Nacional de Universidades (CNU) (1998). Informe Estadístico 1995. 1996, Managua, Nicaragua: Autor.

Durán, María de los Ángeles (comp.) (1982). Liberación y Utopia. Madrid, España: Akal.

Ellsberg, Mary et al. (1998). Confites en el Infierno. Managua, Nicaragua: UNAN/Red de Mujeres Contra la Violencia/Universidad Umea de Suecia.

Haraway, Donna J. (1995). Ciencia, cyborgs y mujeres. Madrid, España: Cátedra. 
Harding, Sara (1996). Ciencia y Feminismo. Madrid, España: Morata.

Instituto Nacional de Estadísticas y Censos/INEC, 1995 (1998). Estadísticas Nacionales, Vol. II. Managua, Nicaragua:Autor.

Keller, Evelyn Fox (1991). Reflexiones sobre Género y Ciencia. Valencia, España: Alfons El Magnánim

Moreno, Amparo (1994). El arquetipo viril protagonista de la historia: Ejercicios para una lectura no androcentrica. Barcelona: Ediciones La Sal.

Montecino, Sonia y Obach, Alexandra (comp.) (1998). Género y Epistemologia: Mujeres y Disciplinas. Santiago, Chile: Programa Interdisciplinario de Estudios de Género/Facultad de Ciencias Sociales/Universidad de Chile.

Mujeres por la Dignidad y la Vida (Las Dignas) (1998). Yo sexista. Material de Apoyo para una Educación No Sexista. San Salvador, El Salvador: Autor.

Programa de las Naciones Unidas para el Desarrollo/PNUD (1999). Informe de Desarrollo Humano 1999 (Breve Reseña). Managua, Nicaragua: Autor.

Renzi, Maria Rosa y Agurto, Sonia (1997). La esperanza tiene nombre de mujer. La economía nicaragüense desde una perspectiva de género. Managua, Nicaragua: FIDEG.

Subirats, Marina (1991). Panorama sobre la situación educativa de las mujeres: Análisis y Políticas. En Lola Luna (comp.), Mujeres y Sociedad. Nuevos enfoques teóricos y metodológicos. Barcelona, España: Seminario Interdisciplinar Mujeres y Sociedad/Universidad de Barcelona.

Universidad Nacional Autónoma de Nicaragua (1998). La UNAN en Cifras 1998. Managua, Nicaragua: UNAN.

Valdés, Teresa y Gomáriz, Enrique (coord.) (1995). Mujeres Latinoamericanas en Cifras. Santiago, Chile: FLACSO/Instituto de la Mujer de España. 